\title{
Attenuation of the Model Error in Observer-Based State-Feedback Regulators
}

\author{
L. Keviczky, Cs. Bányász \\ Széchenyi István University, Győr \\ Computer and Automation Research Institute and Control Engineering Research \\ Group, Hungarian Academy of Sciences \\ H-1111 Budapest, Kende u 13-17, Hungary \\ E-mail: keviczky@sztaki.hu, banyasz@sztaki.hu
}

Abstract: An equivalent transfer function representation (TFR) is introduced to study the state-feedback/observer (SFO) topologies of control systems. This approach is used to explain why an observer can radically reduce even large model errors. Then the same principle is combined with Youlaparametrization (YP) introducing a new class of regulators.

Keywords: observer, state-feedback, model error, Youla-parametrization

\section{Introduction}

It is a well-known methodology to use the state variable representations (SVR) of linear time invariant (LTI) single input - single output (SISO) systems. The SVR proved to be excellent tool to implement both LQR (Linear system - Quadratic criterion - Regulator) control and pole placement design. The practical applicability required to introduce the observers, which make this methodology widely applied even for large scale and higher dimension plants [1]. The thousands of theoretical considerations mostly concentrate on the irregularities and special structures in the SVR appearing. Much less publications deal with the model error properties of these systems.

It is possible to find a proper way to discuss and investigate the limitations if someone replaces the SVR by their TFR. The paper first summarizes the classical state-feedback (SF), state-feedback/observer (SFO) topologies then introduces the $\mathrm{TF}$ equivalent forms. Then the model error properties are discussed and it is shown why the SFO method reduces these errors comparing to a trivial parallel model approach. Finally this principle is extended for Youla-parametrized controllers. 


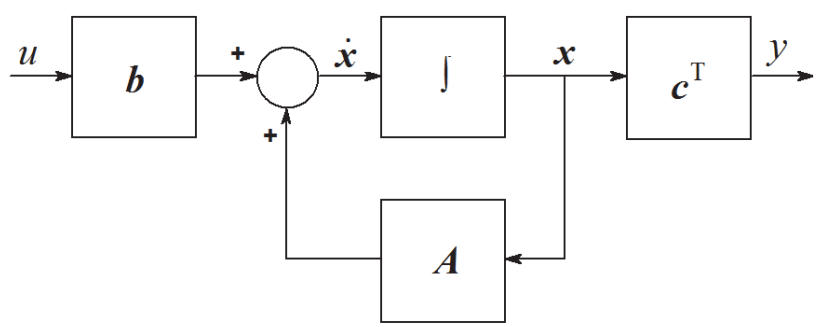

Figure 1. Block diagram of the SVR of a SISO dynamic LTI system

\section{State feedback (SF)}

Consider a SISO continuous time $(t)$ LTI dynamic plant described by the SVR

$$
\begin{gathered}
\frac{\mathrm{d} x}{\mathrm{~d} t}=\dot{\boldsymbol{x}}=\boldsymbol{A} \boldsymbol{x}+\boldsymbol{b} u \\
y=\boldsymbol{c}^{T} \boldsymbol{x}
\end{gathered}
$$

The corresponding block diagram of the process is shown in Fig. 1. Here $u, y$ and $\boldsymbol{x}$ are the input, output and state variables of the process to be controlled and $\mathrm{T}$ stands for transposition.

The TFR of the open-loop system can be calculated by

$$
P=\frac{\mathrm{B}}{\mathrm{A}}=\boldsymbol{c}^{\mathrm{T}}(s \boldsymbol{I}-\boldsymbol{A})^{-1} \boldsymbol{b}
$$

where $\boldsymbol{I}$ is the unit matrix and

$$
\begin{aligned}
& B(s)=s^{n}+b_{1} s^{n-1}+\cdots+b_{n-1} s+b_{n} \\
& A(s)=s^{n}+a_{1} s^{n-1}+\cdots+a_{n-1} s+a_{n}
\end{aligned}
$$

are the numerator and denominator polynomials, respectively. If the feedback is restricted to a linear SF, then the classical solution can be written as

$$
u=k_{\mathrm{r}} r-\boldsymbol{k}^{\mathrm{T}} \boldsymbol{x}
$$

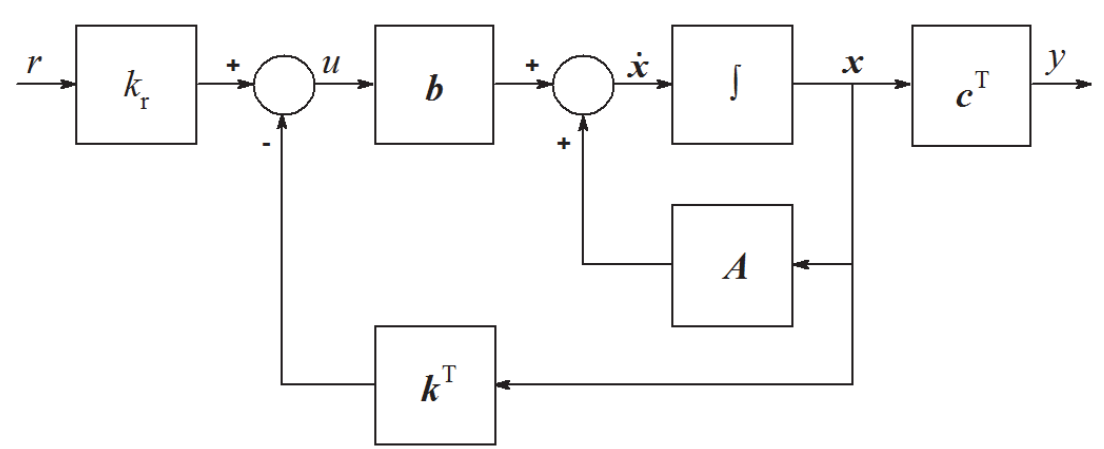

Figure 2. Block diagram of the SF of a SISO LTI system 
The resulting closed-loop system is shown in Fig. 2, where $r$ is the reference signal, $k_{r}$ is a calibrating constant and $\boldsymbol{k}^{\mathrm{T}}$ is the linear SF vector. It is easy to check that the complementary sensitivity function (CSF) from the reference signal $r$ to the output $y$ is

$$
T_{\mathrm{ry}}(s)=k_{\mathrm{r}} \boldsymbol{c}^{\mathrm{T}}\left(s \boldsymbol{I}-\boldsymbol{A}+\boldsymbol{b} \boldsymbol{k}^{\mathrm{T}}\right)^{-1} \boldsymbol{b}=\frac{k_{\mathrm{r}} P}{1+\boldsymbol{k}^{\mathrm{T}}(s \boldsymbol{I}-\boldsymbol{A})^{-1} \boldsymbol{b}}
$$

where $k_{r}$ is obtained by requiring that the static gain of $T_{\mathrm{ry}}$ should be equal to one

$$
k_{\mathrm{r}}=\frac{\boldsymbol{k}^{\mathrm{T}} \boldsymbol{A}^{-1} \boldsymbol{b}-1}{\boldsymbol{c}^{\mathrm{T}} \boldsymbol{A}^{-1} \boldsymbol{b}}
$$

The calibrating factor $k_{r}$ is necessary because the closed-loop using SF is not an integrating one.

The usual classical design goal is to determine the feedback gain $\boldsymbol{k}^{\mathrm{T}}$ so that the closedloop system has the characteristic polynomial

$$
R(s)=s^{n}+r_{1} s^{n-1}+\cdots+r_{n-1} s+r_{n}
$$

The solution formally means making the characteristic polynomial of the closed-loop equal to the desired polynomial ("placed poles")

$$
\operatorname{det}\left(s \boldsymbol{I}-\boldsymbol{A}+\boldsymbol{b} \boldsymbol{k}^{\mathrm{T}}\right)=\mathrm{R}(s)
$$

The solution always exists if $P$ is controllable. If the TFR of the process is known then one can easily form a controllable canonical form with

$$
A_{\mathrm{c}}=\left[\begin{array}{ccccc}
-a_{1} & -a_{2} & \cdots & -a_{n-1} & -a_{n} \\
1 & 0 & \cdots & 0 & 0 \\
0 & 1 & & 0 & 0 \\
\vdots & \vdots & & \vdots & \vdots \\
0 & 0 & 0 & 1 & 0
\end{array}\right] ; \boldsymbol{c}_{\mathrm{c}}^{\mathrm{T}}=\left[b_{1}, b_{2}, \cdots, b_{n}\right] ; \quad \boldsymbol{b}_{\mathrm{c}}=[1,0, \cdots, 0]^{\mathrm{T}}
$$

and the feedback gain is obtained from

$$
\boldsymbol{k}_{\mathrm{c}}^{\mathrm{T}}=\left[r_{1}-a_{1}, r_{2}-a_{2}, \cdots, r_{n}-a_{n}\right]
$$

furthermore the calibration factor is calculated by

$$
k_{\mathrm{r}}=\frac{a_{n}+\left(r_{n}-a_{n}\right)}{b_{n}}=\frac{r_{n}}{b_{n}}
$$

The SVR of the closed-loop system is described by

$$
\begin{aligned}
\frac{\mathrm{d} \boldsymbol{x}}{\mathrm{d} t} & =\left(\boldsymbol{A}-\boldsymbol{b} \boldsymbol{k}^{\mathrm{T}}\right) \boldsymbol{x}+k_{\mathrm{r}} \boldsymbol{b} r \\
y & =\boldsymbol{c}^{\mathrm{T}} \boldsymbol{x}
\end{aligned}
$$


It is easy to see from equation (13) that $T_{\mathrm{ry}}(s)$ is now

$$
T_{\mathrm{ry}}(s)=\frac{k_{\mathrm{r}} \mathrm{B}(s)}{\mathrm{R}(s)}
$$

i.e., besides reaching the desired pole-placement the SF leaves the open-loop zeros untouched. If the TFR is not known and we must use a general SVR then the controller gain is obtained as [2]

$$
\boldsymbol{k}^{\mathrm{T}}=\boldsymbol{k}_{\mathrm{c}}^{\mathrm{T}} \boldsymbol{M}_{\mathrm{c}}^{\mathrm{c}} \boldsymbol{M}_{\mathrm{c}}^{-1}
$$

where $\boldsymbol{M}_{\mathrm{c}}$ is the controllability matrix

$$
M_{\mathrm{c}}=\left[\boldsymbol{b}, A b, \cdots, A^{n-1} b\right]
$$

and $\boldsymbol{M}_{c}^{c}$ is the controllability (Vandermunde) matrix of the controllable canonical form

$$
\boldsymbol{M}_{\mathrm{c}}^{\mathrm{c}}=\left[\begin{array}{ccccc}
1 & a_{1} & a_{2} & \cdots & a_{n-1} \\
0 & 1 & a_{1} & \cdots & a_{n-2} \\
\vdots & \vdots & \vdots & & \vdots \\
0 & 0 & 0 & \cdots & a_{1} \\
0 & 0 & 0 & \cdots & 1
\end{array}\right]^{-1}
$$

(Note that there are several other methods available for calculating the optimal controller gain.)

If we want to express the operation of the SF by equivalent scheme using TFR forms Fig. 3 can be used, where the feedback regulator $R_{f}=K_{k}$ is obtained from the basic equation of the closed-loop

$$
T_{\mathrm{ry}}(s)=\frac{k_{\mathrm{r}} \mathrm{B}(s)}{\mathrm{R}(s)}=\frac{k_{\mathrm{r}} \mathrm{B}(s)}{\mathrm{A}(s)+\mathrm{K}(s)}=\frac{k_{\mathrm{r}} P}{1+K_{k} P}
$$

which clearly shows, that the open-loop poles remain unchanged and the closed-loop poles will be the required ones. Here it is obtained that

$$
R_{\mathrm{f}}=K_{k}(s)=\frac{\mathrm{K}(s)}{\mathrm{B}(s)}=\frac{\mathrm{R}(s)-\mathrm{A}(s)}{\mathrm{B}(s)}=\frac{\boldsymbol{k}^{\mathrm{T}}(s \boldsymbol{I}-\boldsymbol{A})^{-1} \boldsymbol{b}}{\boldsymbol{c}^{\mathrm{T}}(s \boldsymbol{I}-\boldsymbol{A})^{-1} \boldsymbol{b}}
$$

and the calibration factor again

$$
k_{\mathrm{r}}=\frac{\boldsymbol{k}^{\mathrm{T}} \boldsymbol{A}^{-1} \boldsymbol{b}-1}{\boldsymbol{c}^{\mathrm{T}} \boldsymbol{A}^{-1} \boldsymbol{b}}=\frac{1+K_{k}(0) P(0)}{P(0)}
$$




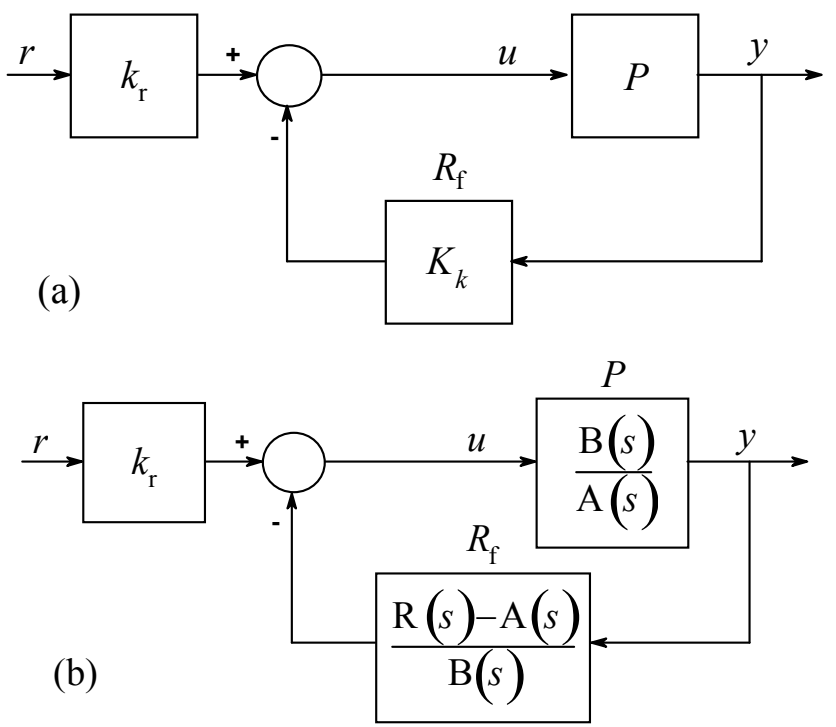

Figure 3. Equivalent schemes of SF using TFR forms

It is a not very frequently discussed question using SF whether the stability of the process polynomials in $P$ are required for the closed-loop stability or not. Figure $3 \mathrm{~b}$ shows the real operation of the SF. The polynomial $R(s)-A(s)$ in the feedback path stabilizes the $1 / A(s)$ denominator of the process, even if it is an unstable one, and places the required poles via the design polynomial $R(s)$. The numerator $B(s)$ of the plant is outside of this process and remains unchanged. Observe that this regulation can be interpreted and realized only if SF is used. Figure 3a shows another interpretation of the controller using only TFR's. This equivalent scheme, however, can be realized by the indicated transfer functions, only if the process $P$ itself is inverse stable (IS), i.e., if $\mathrm{B}(\mathrm{s})$ is a stable polynomial. So note that $K_{k}(s)$ is realizable for IS process and can be used for stabilization if the process is unstable.

The final conclusion is that the SF stabilizes all observable plants, however, the zeros of the process remain unchanged. Further compensation is necessary if we want to handle these zeros. Note that it is very rare that all state variables are measurable in case of a real plant. 


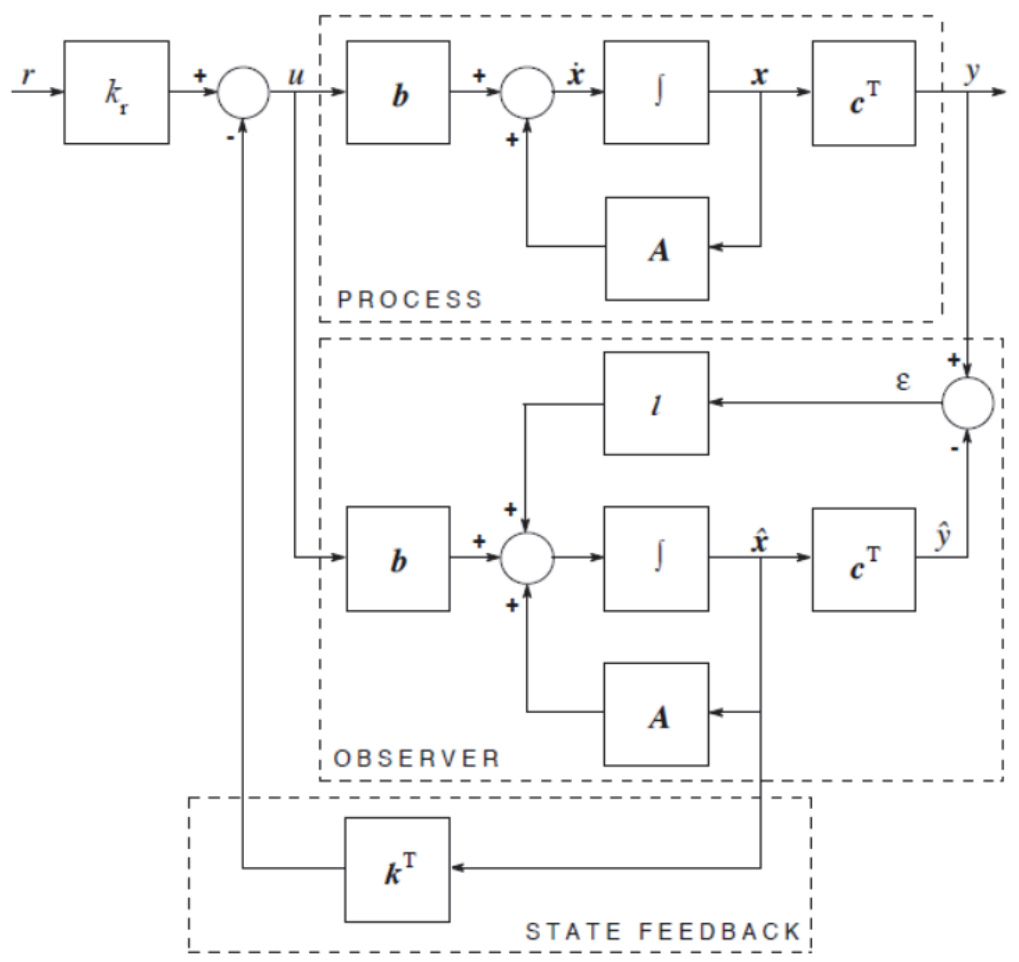

Figure 4. The general basic SFO scheme

\section{Observer-Based State-Feedback}

The practical applicability of the SF theory was introduced by the development of the observers capable to calculate the unmeasured state variables. The most general SF/observer (SFO) topology is shown in Fig. 4.

In the general basic SFO scheme the controller consists of two parts: one observer and one SF. The observer calculates the estimated state variable $\hat{x}$ and the estimated process output $\hat{y}$. The feedback gain $\boldsymbol{k}^{\mathrm{T}}$ is computed, as if all state variables could be measured, using $\hat{x}$, so

$$
u=k_{\mathrm{r}} r-\boldsymbol{k}^{\mathrm{T}} \hat{\boldsymbol{x}}
$$

The observer modifies the internal model of the process by introducing a proportional feedback $\boldsymbol{k}$ from the error $\varepsilon=y-\hat{y}$. It is long and not easy derivation to prove that the $\mathrm{CSF}$ from the reference signal $r$ to the output $y$ is

$$
T_{\mathrm{ry}}(s)=\frac{k_{\mathrm{r}} \boldsymbol{c}^{\mathrm{T}}(s \boldsymbol{I}-\boldsymbol{A})^{-1} \boldsymbol{b}}{1+\boldsymbol{k}^{\mathrm{T}}(s \boldsymbol{I}-\boldsymbol{A})^{-1} \boldsymbol{b}}=\frac{k_{\mathrm{r}} P}{1+\boldsymbol{k}^{\mathrm{T}}(s \boldsymbol{I}-\boldsymbol{A})^{-1} \boldsymbol{b}}=k_{\mathrm{r}} \mathrm{B}(s) / \mathrm{R}(s)
$$


thus surprisingly exactly the same as it was for the simple SF case (see equation (6) and [3] for a nice short derivation). This means that the tracking properties of the SFO do not depend on the selected observer gain $\boldsymbol{l}$. (Note that this is only for the exact knowledge of the process parameters in the observer.) This means that the calculation of the calibration factor is also the same. It is possible to compute an equivalent feedback regulator in this case, too

$$
R_{\mathrm{f}}=\boldsymbol{k}^{\mathrm{T}}\left(s \boldsymbol{I}-\boldsymbol{A}+\boldsymbol{b} \boldsymbol{k}^{\mathrm{T}}+\boldsymbol{l} \boldsymbol{c}^{\mathrm{T}}\right)^{-1} \boldsymbol{l}=\frac{\boldsymbol{k}^{\mathrm{T}}\left(s \boldsymbol{I}-\boldsymbol{A}+\boldsymbol{b} \boldsymbol{k}^{\mathrm{T}}\right)^{-1} \boldsymbol{l}}{1+\boldsymbol{c}^{\mathrm{T}}\left(s \boldsymbol{I}-\boldsymbol{A}+\boldsymbol{b} \boldsymbol{k}^{\mathrm{T}}\right)^{-1} \boldsymbol{l}}
$$

which has a much more complex structure than what was in (19).

Introducing the state error

$$
\tilde{\boldsymbol{x}}=\boldsymbol{x}-\hat{\boldsymbol{x}}
$$

the dynamics of the observer is basically determined by the state error equation

$$
\frac{\mathrm{d} \tilde{\boldsymbol{x}}}{\mathrm{d} t}=\left(\boldsymbol{A}-\boldsymbol{k} \boldsymbol{c}^{\mathrm{T}}\right) \hat{\boldsymbol{x}}
$$

which formally very similar to (13) with no excitation. The usual classical design goal for the observer is to determine the observer feedback gain $\boldsymbol{k}$ so that the dynamic system (25) has the characteristic polynomial

$$
Q(s)=s^{n}+q_{1} s^{n-1}+\cdots+q_{n-1} s+q_{n}
$$

The solution formally means making the characteristic polynomial equal to the desired polynomial

$$
\operatorname{det}\left(s \boldsymbol{I}-\boldsymbol{A}+\boldsymbol{l} \boldsymbol{c}^{\mathrm{T}}\right)=\mathrm{Q}(s)
$$

The solution always exists if $P$ is observable. If the TFR of the process is known then one can easily form an observable canonical form with

$$
\boldsymbol{A}_{\mathrm{o}}=\left[\begin{array}{ccccc}
-a_{1} & 1 & 0 & \cdots & 0 \\
-a_{2} & 0 & 1 & \cdots & 0 \\
\vdots & \vdots & \vdots & & \vdots \\
-a_{n-1} & 0 & 0 & \cdots & 1 \\
-a_{n} & 0 & 0 & \cdots & 0
\end{array}\right] ; c_{\mathrm{o}}^{\mathrm{T}}=[1,0, \cdots, 0] ; \boldsymbol{b}_{\mathrm{o}}=\left[b_{1}, b_{2}, \cdots, b_{n}\right]^{\mathrm{T}}
$$

and the feedback gain is obtained from

$$
\boldsymbol{l}_{\mathrm{o}}=\left[q_{1}-a_{1}, q_{2}-a_{2}, \cdots, q_{n}-a_{n}\right]^{\mathrm{T}}
$$

If the TFR is not known and we must use a general SVR then the observer gain is obtained as [2] 


$$
\boldsymbol{l}=\boldsymbol{M}_{\mathrm{o}}^{-1} \boldsymbol{M}_{\mathrm{o}}^{\mathrm{o}} \boldsymbol{l}_{\mathrm{o}}
$$

where $\boldsymbol{M}_{o}$ is the observability matrix

$$
\boldsymbol{M}_{\mathrm{o}}=\left[\boldsymbol{c}^{\mathrm{T}}, \boldsymbol{c}^{\mathrm{T}} \boldsymbol{A}, \cdots, \boldsymbol{c}^{\mathrm{T}} \boldsymbol{A}^{n-1}\right]^{\mathrm{T}}
$$

and $\boldsymbol{M}_{o}^{o}$ is the observability (Vandermunde) matrix of the observable canonical form

$$
\boldsymbol{M}_{\mathrm{o}}^{\mathrm{o}}=\left[\begin{array}{lllll}
1 & 0 & \cdots & 0 & 0 \\
a_{1} & 1 & \cdots & 0 & 0 \\
a_{2} & a_{1} & \cdots & 0 & 0 \\
\vdots & \vdots & & 1 & 0 \\
a_{n-1} & a_{n-2} & \cdots & a_{1} & 1
\end{array}\right]^{-1}
$$

(Note that there are several methods available for calculating the optimal observer gain.)

There exists an obvious duality between finding the SF and the observer applying the following equivalence: $\boldsymbol{A} \leftrightarrow \boldsymbol{A}^{T}, \boldsymbol{b} \leftrightarrow \boldsymbol{c}^{T}, \boldsymbol{k} \leftrightarrow \boldsymbol{l}^{T}$ and $\boldsymbol{M}_{c}^{c} \leftrightarrow\left(\boldsymbol{M}_{o}^{o}\right)^{T}$.

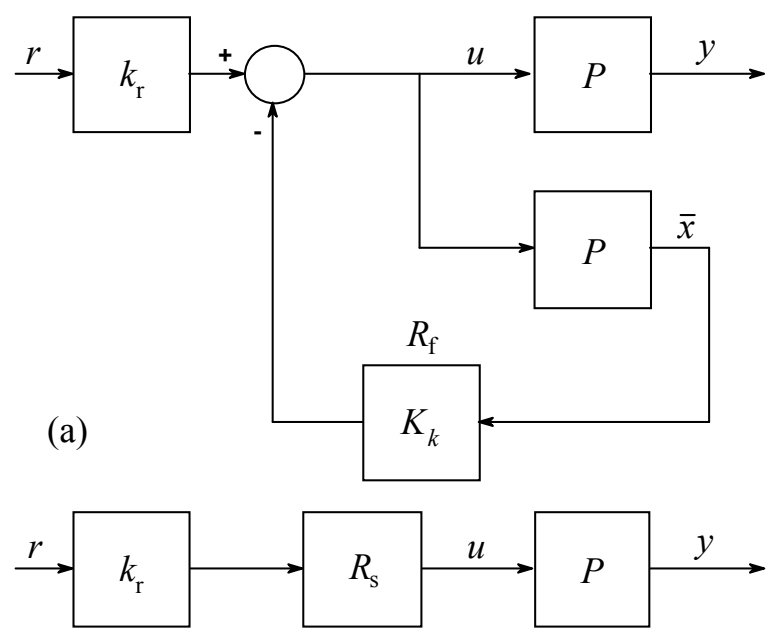

(b)

Figure 5. Equivalent schemes of SF using TFR forms

The SVR of the entire SFO closed-loop system is described by

$$
\begin{aligned}
\frac{\mathrm{d}}{\mathrm{d} t}\left[\begin{array}{l}
\boldsymbol{x} \\
\tilde{\boldsymbol{x}}
\end{array}\right] & =\left[\begin{array}{cc}
\boldsymbol{A}-\boldsymbol{b} \boldsymbol{k}^{\mathrm{T}} & \boldsymbol{b} \boldsymbol{k}^{\mathrm{T}} \\
\mathbf{0} & \boldsymbol{A}-\boldsymbol{l} \boldsymbol{c}^{\mathrm{T}}
\end{array}\right]\left[\begin{array}{l}
\boldsymbol{x} \\
\tilde{\boldsymbol{x}}
\end{array}\right]+\left[\begin{array}{c}
\boldsymbol{k}_{\mathrm{r}} \boldsymbol{b} \\
\mathbf{0}
\end{array}\right] r \\
e & =y-\hat{y}=\boldsymbol{c}^{\mathrm{T}} \tilde{\boldsymbol{x}}
\end{aligned}
$$


Since the matrix on the right-hand side is block diagonal the characteristic equation of the closed loop system is

$$
\operatorname{det}\left(s \boldsymbol{I}-\boldsymbol{A}+\boldsymbol{b} \boldsymbol{k}^{\mathrm{T}}\right) \operatorname{det}\left(s \boldsymbol{I}-\boldsymbol{A}+\boldsymbol{l} \boldsymbol{c}^{\mathrm{T}}\right)=\mathrm{R}(s) \mathrm{Q}(s)
$$

This polynomial is the product of two terms: one which is used for the $S F$ design and the other which is used for the observer design. In spite of (34) it is interesting to observe that $\mathrm{Q}(s)$ does not appear in the $\mathrm{T}_{\mathrm{ry}}(s)$ given by $(22)$. The explanation of this phenomenon can be given by the investigation of the internal topology of the equivalent TFR forms of the $S F O$ scheme.

\section{Equivalent TFR Forms of the SFO Scheme}

Introducing Figs. 3a-b the technique using equivalent TFR forms of $S F$ has been discussed above. To get a more general procedure consider Figs. 3a-b again in the forms presented in Figs. 5a-b. It follows from Fig. 5 that the serial compensator $R_{s}$ can be calculated by

$$
R_{\mathrm{S}}=\frac{1}{1+R_{\mathrm{s}} P}=\frac{1}{1+K_{k} P}=\frac{\mathrm{A}(s)}{\mathrm{A}(s)+\mathrm{K}(s)}=\frac{\mathrm{A}(s)}{\mathrm{R}(s)}
$$

Observe that this serial compensator cannot be applied for unstable processes because of the full pole cancellation in $R_{s}$, in spite the fact that $k_{r} R_{S} P$ ensures the same overall transfer function $T_{\mathrm{ry}}$. Finding the equivalent TFR form an auxiliary internal signal $\bar{x}$ is introduced and used (which is not equal to $\boldsymbol{x}$ ) indicating that finally both the $S F$ and the observer use a SISO filter realizing their effect. The difference is that they use internal state variable vectors $(\boldsymbol{x}, \widehat{\boldsymbol{x}}, \widetilde{\boldsymbol{x}}$ etc.) instead of scalar ones. It is always possible to find input/output equivalence between these representations. Using this approach the general basic $S F O$ scheme in Fig. 4 can be redrawn into another topology shown in Fig. 6.

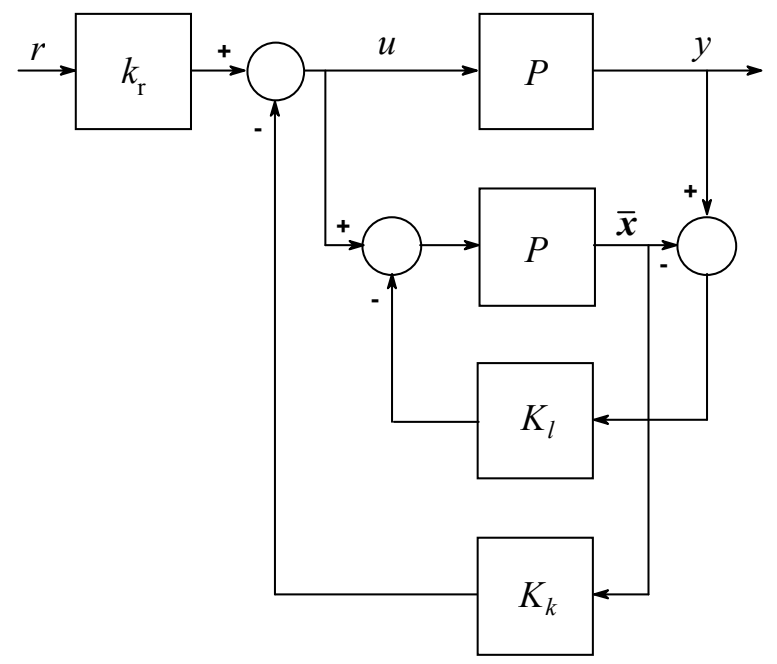

Figure 6. Equivalent topology of the general basic SFO scheme using TFR forms 
After some long, but straightforward block manipulations the equivalent $S F O$ scheme can be transformed into another unity feedback closed-loop form given in Fig. 7.

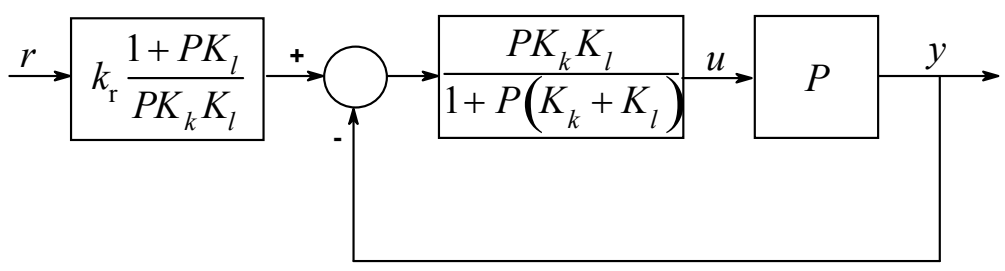

Figure 7. Reduced equivalent topology of the general basic SFO scheme

Here reasonably and in accordance with the previous discussions the following TFR forms are selected:

$$
K_{k}(s)=\frac{\mathrm{K}(s)}{\mathrm{B}(s)} \quad K_{l}(s)=\frac{\mathrm{L}(s)}{\mathrm{B}(s)}
$$

where the pole-placement design goals for both the $S F$ and the observer dynamics require

$$
\mathrm{K}(s)=\mathrm{R}(s)-\mathrm{A}(s) \quad \mathrm{L}(s)=\mathrm{Q}(s)-\mathrm{A}(s)
$$

It is interesting to observe that the transfer function of the closed-loop in Fig. 7 has a very special structure

$$
\frac{P^{2} K_{k} K_{l}}{1+P\left(K_{k}+K_{l}\right)+P^{2} K_{k} K_{l}}=\frac{P K_{k}}{1+P K_{k}} \frac{P K_{l}}{1+P K_{l}}=\frac{\mathrm{K}}{\mathrm{R}} \frac{\mathrm{L}}{\mathrm{Q}}
$$

which is formally two simpler closed-loop cascaded, which dynamically completely corresponds to the characteristic equation (34). The overall transfer function of the SFO system is

$$
T_{\mathrm{ry}}(s)=k_{\mathrm{r}} \frac{1+P K_{l}}{P K_{k} K_{l}} \frac{P K_{k}}{1+P K_{k}} \frac{P K_{l}}{1+P K_{l}}=\frac{k_{\mathrm{r}} P}{1+P K_{k}}=\frac{k_{\mathrm{r}} \mathrm{B}}{\mathrm{R}}
$$

which is equal to (22) as expected: the poles introduced by the observer do not appear in the tracking dynamics of the $S F O$ system. This behaviour can be well seen in Fig. 8 .

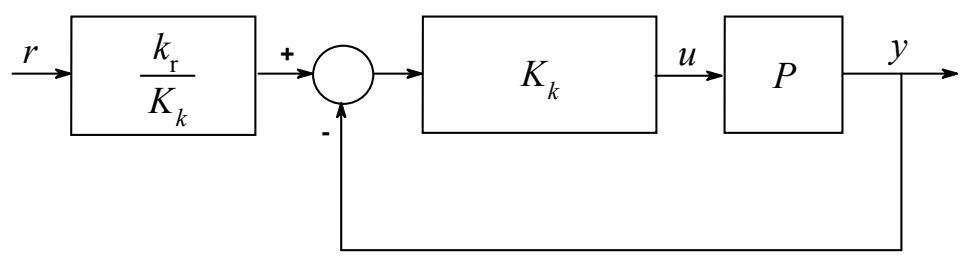

Figure 8. The overall tracking structure of the SFO scheme 


\section{Model Error Properties}

The above widely applied methodology has a common problem, that in all regulator and observer equations the true process $P$ is used instead of the estimated model $\hat{P}$ of the process. A logical notation should be if $\widehat{\boldsymbol{A}}, \widehat{\boldsymbol{b}}, \hat{\boldsymbol{c}}^{T}$, belonging to $\hat{P}$ is used instead of $\boldsymbol{A}, \boldsymbol{b}, \boldsymbol{c}^{\mathrm{T}}$, representing $P$. The equivalent $T F R$ form of the $S F$ using the model of the process is shown in Fig. 9 .

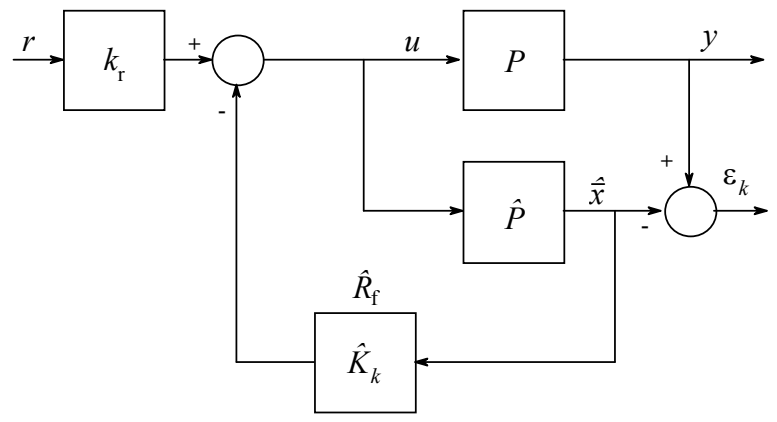

Figure 9. The model based SF scheme and error

In spite of the practical reality of this scheme a major drawback raises with the parallel model TFR of the $S F$ method, because it cannot stabilize unstable plants. A model-based equivalent serial compensator (35) cannot provide stable operation with pole cancellation. The $S F$ is operable if the exact states are available for feedback. So the parallel scheme in Fig. 9 is used only to compute the model error. Using (22) the model-based version of $T_{\mathrm{ry}}$ is

$$
\hat{T}_{\mathrm{ry}}=\frac{k_{\mathrm{r}} P}{1+K_{k} \hat{P}}=\frac{k_{\mathrm{r}} \mathrm{B}}{\mathrm{R}} \frac{\hat{\mathrm{A}}}{\mathrm{A}}=T_{\mathrm{ry}} \frac{\hat{\mathrm{A}}}{\mathrm{A}}
$$

and its relative uncertainty

$$
\ell_{\mathrm{T}}=\frac{\hat{T}_{\mathrm{ry}}-T_{\mathrm{ry}}}{\hat{T}_{\mathrm{ry}}}=\frac{\hat{\mathrm{A}}-\mathrm{A}}{\mathrm{A}}=\ell_{\mathrm{A}}
$$

which shows that $\ell_{\mathrm{T}}=0$ for $\ell_{A}=0$. Introducing the additive $\Delta=P-\hat{P}$ and relative plant model error

$$
\ell=\frac{\Delta}{\hat{P}}=\frac{P-\hat{P}}{\hat{P}}
$$

the modelling error $\varepsilon_{k}$ in Fig. 9 can be expressed as

$$
\varepsilon_{k}=\frac{k_{\mathrm{r}} \hat{\mathrm{B}}}{\mathrm{R}} \ell r=T_{\mathrm{ry}} \frac{\hat{\mathrm{B}}}{\mathrm{B}} \ell r=\hat{P} \ell u
$$


After some long but straightforward computations

$$
\varepsilon_{l}=\frac{\hat{P}}{1+K_{l} \hat{P}} \ell u=\frac{\hat{\mathrm{B}}}{\mathrm{Q}} \ell u=\frac{1}{1+K_{l} \hat{P}} \varepsilon_{k}
$$

is obtained. Equation (44) clearly shows the influence of the $S F O$ scheme, because it decreases the modelling error $\varepsilon_{k}$ by $\left(1+K_{l} \hat{P}\right)$. Selecting fast observer poles, one can reach quite small "virtual" modelling error $\varepsilon_{l}$ in the major frequency domains of the tracking task.

In spite of the above analysis the $S F O$ scheme is widely applied in the practice with model-based $S V R$, so it is interesting how the model-based scheme in Fig. 10 influences the original modelling error $\varepsilon_{k}$.

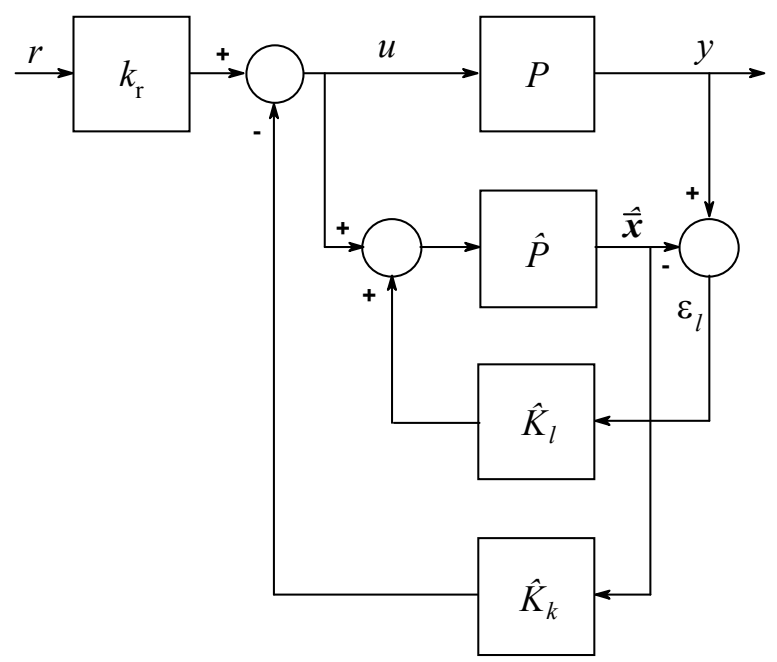

Figure 10. Model based SFO scheme with TFR forms

Besides the radical model error attenuating behaviour of the model-based SFO scheme, it has also a very important drawback, namely the nice cascade structure shown in (38) changes to

$$
\frac{\hat{P}^{2} K_{k} K_{l}(1+\ell)}{1+\hat{P}\left(K_{k}+K_{l}\right)+\hat{P}^{2} K_{k} K_{l}(1+\ell)}
$$

which form is not factorable. On the basis of Fig. 10 and (45) it is easy to see that the poles of the observer feedback loop remains unchanged using the placement design equation form model-based SFO (29). However, in this case the pole placement equation (11) is no longer valid. The only solution is to use the available model of the process, in this case $\hat{A}$, and

$$
\hat{\boldsymbol{k}}_{\mathrm{c}}^{\mathrm{T}}=\left[p_{1}-\hat{a}_{1}, p_{2}-\hat{a}_{2}, \ldots, p_{n}-\hat{a}_{n}\right]
$$


for the pole placing equation. Because this design ensures the required poles only for small $\ell$ (see (46), therefore a serious robust stability investigation is necessary first. Next it is important where the actual pole can be located for not zero $\ell$, so how big is the performance loss coming from the model based SFR. These steps are usually neglected in most of the published papers, books and applications.

\section{Observer Based Youla-Regulator}

For open-loop stable processes the all realizable stabilizing $(A R S)$ regulator is the Youla-parametrized one:

$$
C=\frac{Q}{1-Q P}
$$

where the "parameter" $Q$ ranges over all proper $(Q(\omega=\infty)$ is finite), stable transfer functions [5], [6].

It is important to know that the $Y$-parametrized closed-loop with the $A R S$ regulator is equivalent to the well-known form of the so-called Internal Model Control (IMC) principle [6] based structure shown in Fig. 11.

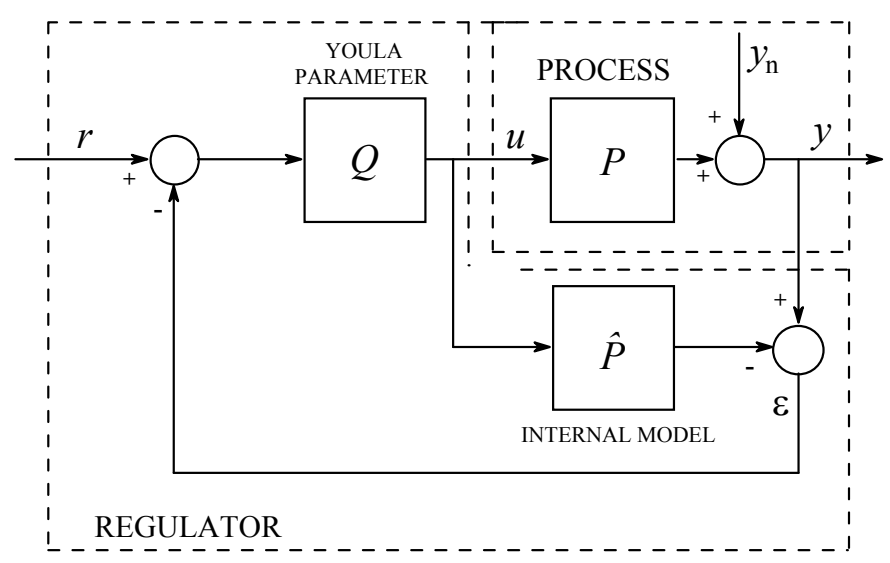

Figure 11. The equivalent IMC structure of an ARS regulator

$Q$ is anyway the transfer function from $r$ to $u$ and the closed-loop transfer function (i.e., $C S F$ ) for $\hat{P}=P$

$$
T_{\text {ry }}=\frac{C P}{1+C P}=Q P
$$

is linear (and hence convex) in $Q$. 


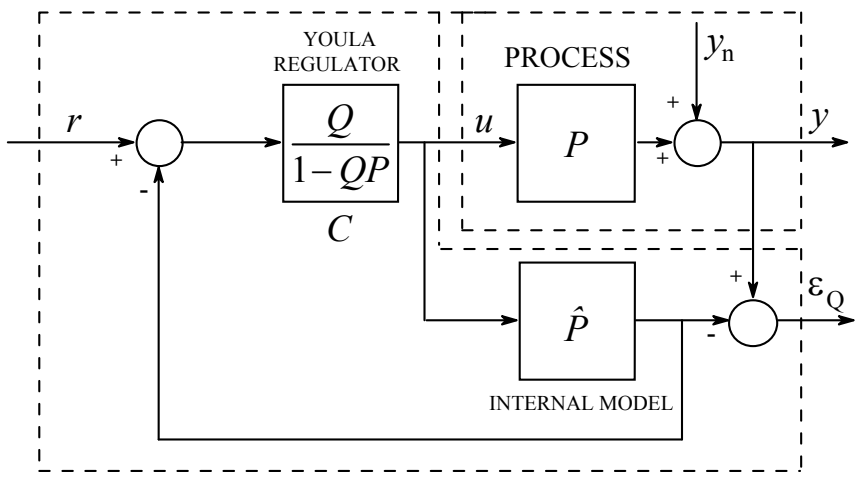

Figure 12. Another equivalent IMC structure

The equivalent $I M C$ structure performs the feedback from the model error $\varepsilon$. It is possible to form another formally equivalent scheme (shown in Fig. 12), where the feedback is from the output of the internal model, similarly to the formerly presented SFO topology.

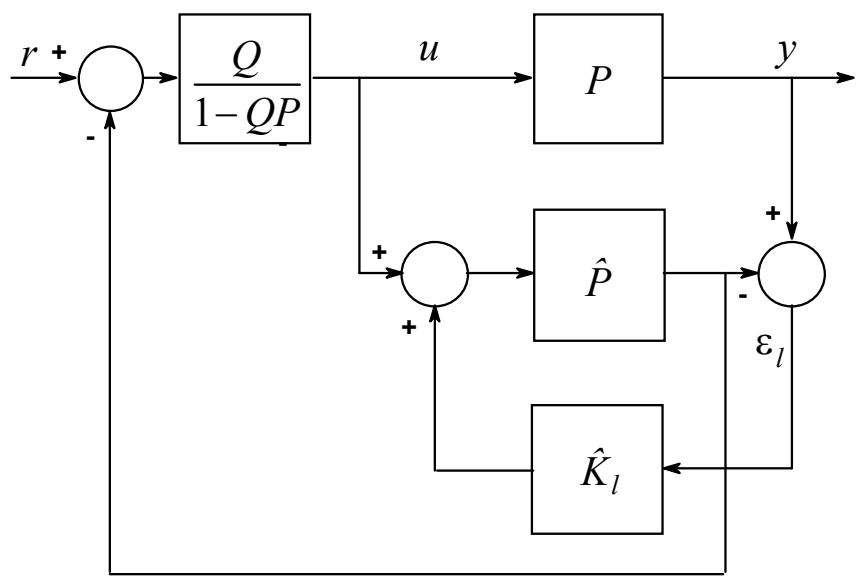

Figure 13. The observer-based Youla-regulator

It is easy to check that the same $T_{\text {ry }}$ can be obtained for this structure in case of $\hat{P}=P$. The advantage of the last equivalent scheme is that it is possible to invent an observerlike feedback, similarly to Fig. 10 as Fig. 13 shows. After some long but straightforward computations

$$
\varepsilon_{l}=\frac{1}{1+\hat{K}_{l} \hat{P}}(y-\hat{P} u)=\frac{1}{1+\hat{K}_{l} \hat{P}} \varepsilon_{Q}
$$

is obtained. Equation (49) clearly shows the influence of the $S F O$ scheme here, too, because it decreases the modelling error $\varepsilon_{Q}$ by $\left(1+\hat{K}_{l} \hat{P}\right)$. Selecting fast observer poles, 
one can reach quite small "virtual" modelling error $\varepsilon_{l}$ in the major frequency domains of the tracking task.

It is not difficult to prove by equivalent block manipulations that the simple closedloop corresponding to the observer-base Youla-regulator has the structure shown in Fig. 14. This means that the introduction of the observer feedback changes the Youlaparametrized regulator to

$$
C^{\prime}=\frac{Q}{1-Q \frac{\hat{P}}{1+\hat{K}_{l} \hat{P}}}=\frac{Q}{1-Q \hat{P}^{\prime}}
$$

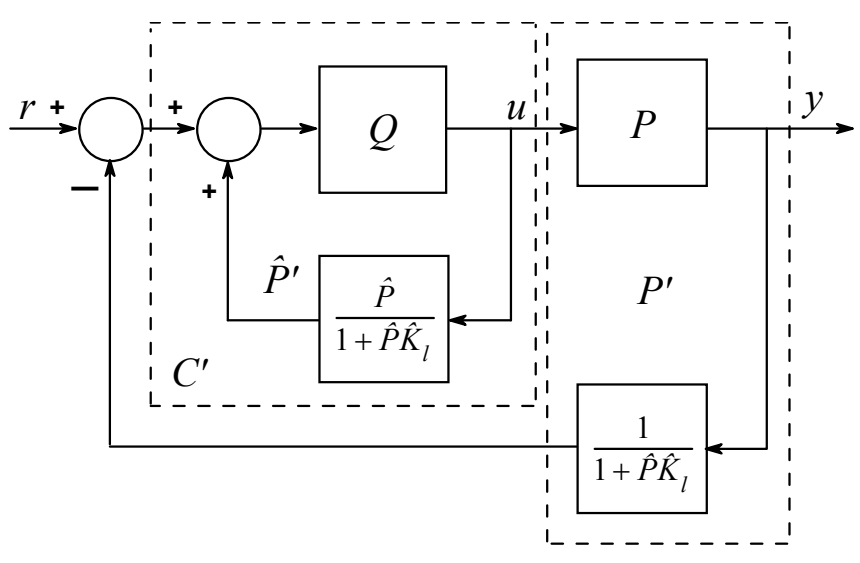

Figure 14 Equivalent closed-loop for the observer-based Youla-regulator

The form of $C^{\prime}$ shows that the regulator virtually controls a fictitious plant $\hat{P}^{\prime}$, which is also demonstrated in Fig. 14. Here the fictitious plant is

$$
\hat{P}^{\prime}=\frac{\hat{P}^{1+\hat{K}_{l} \hat{P}}}{1}
$$

\section{Conclusions}

It was shown that the $S F O$ methodology results in such a $T D O F$ control system, which leaves the open-loop zeros untouched for the tracking properties and unfortunately the disturbance rejection (regulatory) properties can only be partly designed, because they are not independent of the tracking design.

The TFR of these classical methods are introduced to get a simple and useful tool to analyse and explain further behaviours, which are difficult to obtain using $S V R$. Using $T F R$ it was shown, if the $S V R$ used in the $S F O$ scheme is model-based then the original (without observer) model error decreases by the sensitivity function of the observer feedback loop. This model error reducing capability gives the theoretical background of the success of practical model-based $S F O$ applications. 
Finally the $S F O$ method was applied for the Youla $A R S$ regulators opening a new class of methods for open-loop stable processes.

\section{Acknowledgement}

This work was supported in part by the Control Engineering Research Group of the Hungarian Academy of Science, at the Budapest University of Technology and Economics and by the project TAMOP 4.2.2.A-11/1/KONV-2012-2012, at the Széchenyi István University of Győr.

\section{References}

[1] Åström, K.J., Wittenmark, B.: Computer controlled systems: theory and design, Prentice-Hall, Englewood Cliffs, NJ, 1984

[2] Åström, K.J.: Control System Design, lecture notes, University of California, Santa Barbara, 2002

[3] Kailath, T.: Linear Systems, Prentice Hall, Englewood Cliffs, NJ, 1980

[4] Keviczky, L.: Combined identification and control: another way. Invited plenary paper, 5th IFAC Symposium on Adaptive Control and Signal Processing, ACASP'95, Budapest, Hungary, pp. 13-30, 1995

[5] Keviczky, L., Bányász, Cs.: Iterative identification and control design using $K-B$ parametrization, In: Control of Complex Systems, Eds: Åström, K.J., Albertos, P., Blanke, M., Isidori, A., Schaufelberger W., Sanz, R., Springer, pp. 101-121, 2001

[6] Maciejowski, J.M.: Multivariable Feedback Design, Addison Wesley, Boston, 1989 